\title{
Neurobrucellosis: clinical, diagnostic, therapeutic features and outcome. Unusual clinical presentations in an endemic region
}

Authors

Nurgul Ceran ${ }^{1}$

Recai Turkoglu ${ }^{2}$

Ilknur Erdem ${ }^{1}$

Asuman Inan ${ }^{1}$

Derya Engin ${ }^{1}$

Hulya Tireli ${ }^{2}$

Pasa Goktas

${ }^{1}$ Department of Infectious Diseases Haydarpasa

Numune Training and Research Hospital, Istanbul, Turkey

${ }^{2}$ Neurology, Haydarpasa Numune Training and

Research Hospital, Istanbul, Turkey
Submitted on: 03/02/2010 Approved on: 09/28/2010

\section{Correspondence to:} Nurgul Ceran

Harem Iskele Caddesi, $80 / 12$

Uskudar, 34668

Istanbul, Turkey

Phone: +90216 3917120

nurgulceran@hotmail.com

We declare no conflict of interest.

\begin{abstract}
Brucellosis is a zoonotic infection and has endemic characteristics. Neurobrucellosis is an uncommon complication of this infection. The aim of this study was to present unusual clinical manifestations and to discuss the management and outcome of a series of 18 neurobrucellosis cases. Initial clinical manifestations consist of pseudotumor cerebri in one case, white matter lesions and demyelinating syndrome in three cases, intracranial granuloma in one case, transverse myelitis in two cases, sagittal sinus thrombosis in one case, spinal arachnoiditis in one case, intracranial vasculitis in one case, in addition to meningitis in all cases. Eleven patients were male and seven were female. The most prevalent symptoms were headache $(83 \%)$ and fever $(44 \%)$. All patients were treated with rifampicin, doxycycline plus trimethoprim-sulfamethoxazole or ceftriaxone. Duration of treatment (varied 3-12 months) was determined on basis of the CSF response. In four patients presented with left mild sequelae including aphasia, hearing loss, hemiparesis. In conclusion, although mortality is rare in neurobrucellosis, its sequelae are significant. In neurobrucellosis various clinical and neuroradiologic signs and symptoms can be confused with other neurologic diseases. In inhabitants or visitors of endemic areas, neurobrucellosis should be kept in mind in cases that have unusual neurological manifestations.
\end{abstract}

Keywords: neurobrucellosis; serology; diagnosis; neuroimaging.

[Braz J Infect Dis 2011;15(1):52-59] @Elsevier Editora Ltda.

\section{INTRODUCTION}

Human brucellosis is a common infectious disease with 18,000 new cases per year in Turkey. It still remains a common problem in some parts of Turkey because of the consumption of contaminated milk or dairy products made from unpasteurized milk. Brucellosis seropositivity is $2.6-14.4 \%$ among Turkish people. ${ }^{1}$

Brucellosis has a wide clinical polymorphism and almost every organ can be affected during the infection. This infection is primarily a reticuloendothelial system (RES) disease, and the most common site involved is the osteoarticular. Additionally, hematologic system, central nervous system, cardiopulmonary system and genitourinary system can be involved to some extent. ${ }^{2-5}$

The incidence of neurological complications range between $0-25 \%$ in adult patients, it is rarely seen in children., ${ }^{2,7}$ Neurological complications are infrequent but have marked clinical importance for their severity and impor- tant morbidity. Brucella bacteria may affect the nervous system directly or indirectly, as a result of cytokine or endotoxin on the neural tissue. ${ }^{8}$ Cytotoxic $\mathrm{T}$ lymphocytes and microglia activation play an immunopathologic role in this disease. ${ }^{9}$ Some immunological mechanisms operate to produce the demyelinating lesions in the cerebral and spinal cord white matter.

CNS involvement is generally in meningoencephalitis form..$^{5,8,9}$ Development of basal meningitis may lead to lymphocytic pleocytosis, cranial nerve involvement or intracranial hypertension. In diagnosing brucellosis the bacteria isolation from serum and other specimens are the gold standard, but culture positivity may be less than $50 \%$ in many reports. ${ }^{2-5,8,10,11}$

In neurobrucellosis imaging findings may be found in four types; normal, inflammation (abnormal enhancement), white matter changes, and vascular changes., ${ }^{2,4}$ Concerning treatment of brucellosis, monotherapy can cause 
relapses, therefore, combined therapy is recommended. The aim of the study was to present different neurological conditions ranging from meningoencephalitis, spinal arachnoiditis, sagittal sinus thrombosis and transverse myelitis to white matter involvement and raised intracranial pressure and to discuss managing and response to therapy of neurobrucellosis.

\section{MATERIAL AND METHODS}

This prospective study included 18 consecutive patients with neurobrucellosis, diagnosed and treated between 2002 and 2008. Eleven of them were followed up at the infectious diseases clinic and the other seven patients who had neurological disorder-like clinic features were followed up at the neurology clinic. All patients had diagnostic criteria for neurobrucellosis. These were:

1- Signs and symptoms of neurological disease in the absence of other diseases.

2- Bacteria isolation from blood and other body fluids.

3- Standard tube agglutination (STA) titers positivity in serum and/or cerebrospinal fluid (CSF).

4- CSF findings, revealing chronic meningitis (lymphocytic pleocytosis, increased protein level, decreased glucose level). Presence of any of these four criteria was sufficient for the diagnosis., ${ }^{2,5,8,11}$

STA commercial kit was obtained from Pendik Veterinarian Research Institute (Istanbul-Turkey). Antibody positivity $>1 / 160$ in serum, and $>1 / 80$ in CSF, or weekly antibody titer increase was considered diagnostic for brucellosis.

Blood and CSF were cultured in routine aerobic and anaerobic automated Bactec system (Bactec 9120 Becton Dickinson). Brucella species were identified at the Pendik Veterinarian Research Institute.

All cases were evaluated according to their clinical symptoms, neurological and physical signs and CSF analysis, as well as backbone $\mathrm{x}$-rays. Chest $\mathrm{X}$-rays were taken to rule out possible lung tuberculosis. All CSF samples were tested by Gram stain, Ziehl- Nielsen stain and VDRL and serum samples by TPHA. Autoimmune disorders were ruled out in a patient who had cerebral thrombosis.

In all patients, neuroimaging techniques, such as computed topography (CT) and magnetic ressonance imaging (MRI), were performed at the onset of illness and during the follow-up period. In our clinic the follow-up protocol for neurobrucellosis patients is as follows. After initiation of treatment LP is performed in all patients once a month to evaluate CSF response to treatment. Cranial CT/MR were repeated in patients with intracranial lesions with intervals of 3-4 weeks. Duration of therapy was determined according to examination results. During a period of three years after discontinuation of treatment the patients were checked in our outpatient clinic every 3-6 months.

\section{RESULTS}

The clinical and neuroradiological presentations of patients with neurobrucellosis are summarized in Table 1.

Eleven patients were male and seven female. The mean age was $38.11 \pm 18.12$. All patients had diagnostic criteria 1,3 , and 4 listed above. Nine patients, who had positive blood culture for brucellosis met all diagnostic criteria. The cases were classified in two different clinical categories. The first group had meningovascular complications as prominent signs. Eleven patients (cases 8-18) had presented symptoms and signs such as fever, neck stiffness, cranial nerve palsies. These symptoms were considered as a central nervous system infection and they were admitted to the infectious disease clinic. The second clinical group had implied diffuse CNS involvement. In this group, brain or spinal cord involvement were prominent signs. The patients (cases 1-7) had presented with symptoms such as cerebellar dysfunctions, transverse myelitis, neurosensorial deafness, hemiplegia, and aphasia. They were admitted to the neurology clinic. However there were overlaps among the clinical categories.

Twelve patients were admitted with symptoms other than meningitis, although all patients have meningeal involvement. The cranial MR of three patients (case 1, 2 , and 8) that had CNS involvement, white matter lesions (Figure 1) were observed. For these patients differential diagnosis from demyelinating disorders was made. In three patients (case 5, 7, and 17), myelitis was a

Figure 1: White matter lesions on T2 weighted MRI scanning (case 1).

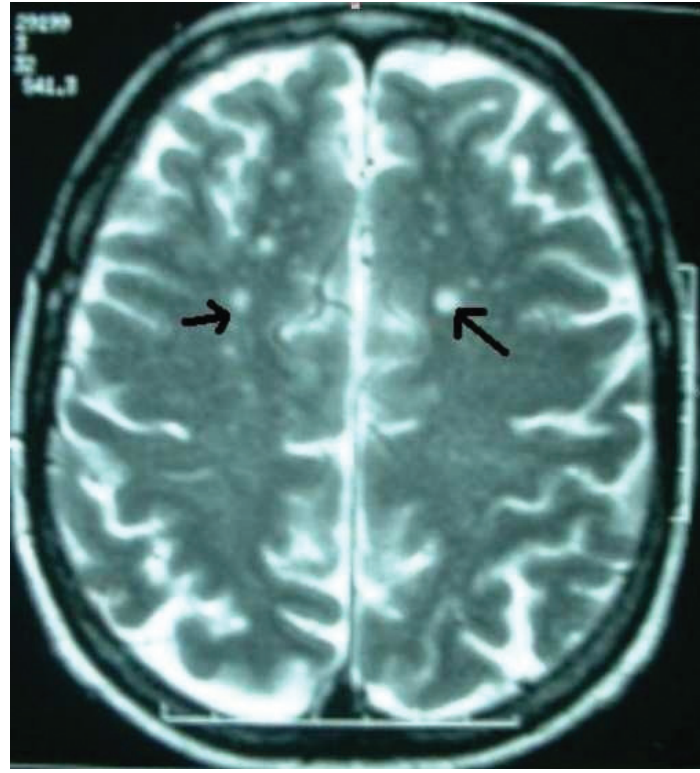


Table 1. Summary of the clinical features and cranial CT/MR of patients with neurobrucellosis

\begin{tabular}{|c|c|c|c|c|c|}
\hline $\mathrm{p} A \mathrm{~S}$ & Neurological & $\begin{array}{c}\text { Diagnosis } \\
\text { symptoms and signs }\end{array}$ & Cranial CT/MRI & $\begin{array}{l}\text { Serum } \\
\text { SAT titers }\end{array}$ & $\begin{array}{c}\text { CSF } \\
\text { SAT titers }\end{array}$ \\
\hline $145 \mathrm{~F}$ & $\begin{array}{l}\text { Papilledema, } \\
\text { intentional tremor }\end{array}$ & Meningoencephalitis & $\begin{array}{l}\text { White matter } \\
\text { lesions (MR) }\end{array}$ & $1 / 640$ & $1 / 320$ \\
\hline $251 \mathrm{M}$ & $\begin{array}{l}\text { Hearing loss (bilateral } \\
\text { neurosensorial auditory } \\
\text { loss), dysmetria, ataxia }\end{array}$ & Meningoencephalitis & $\begin{array}{c}\text { Meningeal contrast (MR) } \\
\text { enhancement white } \\
\text { matter lesions }\end{array}$ & $1 / 320$ & $1 / 160$ \\
\hline $336 \mathrm{M}$ & $\begin{array}{l}\text { Diplopia, right abducens } \\
\text { palsy, motor aphasia, } \\
\text { right hemiparesis }\end{array}$ & Meningoencephalitis & $\begin{array}{l}\text { Meningeal contrast (MR) } \\
\text { enhancement }\end{array}$ & $1 / 320$ & $1 / 160$ \\
\hline $445 \mathrm{~F}$ & $\begin{array}{l}\text { Depression, } \\
\text { papilledema }\end{array}$ & $\begin{array}{c}\text { Meningitis plus } \\
\text { Intracranial hypertension }\end{array}$ & $\begin{array}{c}\text { Meningeal contrast (MR) } \\
\text { enhancement }\end{array}$ & $1 / 640$ & $1 / 160$ \\
\hline $563 \mathrm{~F}$ & $\begin{array}{c}\text { Quadriparesis }(3 / 5) \text {, } \\
\text { bilateral dysesthesia below T2, } \\
\text { urinary and anal incontinans }\end{array}$ & Meningomyelitis & $\begin{array}{c}\text { Spinal MR: cervical } \\
\text { medulla spinalis involvement }\end{array}$ & $1 / 320$ & $1 / 180$ \\
\hline $655 \mathrm{M}$ & $\begin{array}{l}\text { Motor aphasia, } \\
\text { right hemiparesis }\end{array}$ & $\begin{array}{c}\text { Vasculitis } \\
\text { (left MCA infarct) }\end{array}$ & Infarction of left MCA (CT) & $1 / 2560$ & ND \\
\hline $735 \mathrm{~F}$ & $\begin{array}{c}\text { Imbalance, lower } \\
\text { extremity contraction, } \\
\text { micturition disturbances }\end{array}$ & Meningomyelitis & $\begin{array}{c}\text { Pachymeningeal } \\
\text { contrast enhancement } \\
\text { in spinal cord (spinal MR) }\end{array}$ & $1 / 640$ & $1 / 320$ \\
\hline $851 \mathrm{~F}$ & Confusion, quadriparesis & Meningoencephalitis & White matter lesions (MR) & $1 / 320$ & $1 / 160$ \\
\hline $936 \mathrm{M}$ & Confusion & Meningoencephalitis & CT and MR normal & $1 / 640$ & $1 / 160$ \\
\hline $1015 \mathrm{M}$ & Confusion & Meningoencephalitis & Meningealcontrast enhancement & at $1 / 320$ & $1 / 80$ \\
\hline $1122 \mathrm{~F}$ & Diplopia, lower & $\begin{array}{l}\text { Meningoencephalitis } \\
\text { extremity paraparesis }\end{array}$ & $\begin{array}{c}\text { Spinal MR } \\
+ \text { spinal arachnoiditis } \\
\text { inflammation of MS }\end{array}$ & $1 / 320$ & $1 / 80$ \\
\hline $1217 \mathrm{M}$ & $\begin{array}{c}\text { Diplopia, } \\
\text { imbalance,speech } \\
\text { disturbances, left nervous } \\
\text { abducens palsy }\end{array}$ & $\begin{array}{c}\text { Meningoencephalitis } \\
+ \text { intracranial } \\
\text { granuloma }\end{array}$ & $\begin{array}{c}\text { Granuloma in left } \\
\text { temporal lobe, vasculitis }\end{array}$ & $1 / 160$ & $1 / 80$ \\
\hline $1314 \mathrm{M}$ & - & Meningitis & CT and MR normal & $1 / 640$ & $1 / 80$ \\
\hline $1426 \mathrm{~F}$ & - & Meningitis & CT and MR normal & $1 / 640$ & $1 / 160$ \\
\hline $1529 \mathrm{M}$ & $\begin{array}{c}\text { Aphasia, sensory } \\
\text { loss in the upper and } \\
\text { lower extremities, confusion }\end{array}$ & Meningoencephalitis & $\begin{array}{l}\text { Cranial MR venography: } \\
\text { sagittal sinus thrombosis }\end{array}$ & $1 / 320$ & $1 / 80$ \\
\hline $1614 \mathrm{M}$ & - & Meningitis & CT and MR normal & $1 / 640$ & $1 / 160$ \\
\hline $1767 \mathrm{M}$ & $\begin{array}{c}\text { Quadriparesis }(3 / 5), \\
\text { micturition disturbances, } \\
\text { nistagmus }\end{array}$ & Meningomyelitis & CT and MR normal & $\begin{array}{l}\text { SAT(-), } \operatorname{IgM} \\
\text { and } \\
\operatorname{IgG}(+)^{*}\end{array}$ & $\begin{array}{l}\text { SAT(-), IgM } \\
\text { and } \\
\operatorname{IgG}(+)^{*}\end{array}$ \\
\hline $1865 \mathrm{M}$ & - & Meningitis & CT and MR normal & $1 / 1280$ & $1 / 160$ \\
\hline
\end{tabular}

P, patients; A, age; S, sex; MS, Medulla spinalis; ND, not done. 
prominent sign at clinical and radiological examination (Figure 2). In case 4 , who had pseudotumor cerebri presented CSF pressure of $600 \mathrm{cmH}_{2} \mathrm{O}$ at the lumbar puncture. Case 6 had history of aortic valve replacement due to rheumatic heart disease. In his neurological examination, he had right hemiparesis and motor aphasia. The patient was admitted to the infectious disease clinic due to preliminary diagnosis of Brucella endocarditis and neurobrucellosis. Transesophageal echocardiography detected a mass sized $4 \times 3.5 \times 2 \mathrm{~cm}$ in the base of the aorta, which was presumed to be an abscess. He underwent an operation for abscess removal and replacement of aorta valve. In case 10 a swelling was observed in his left parotid on the third day of Brucella treatment. Antimumps IgM was negative. White blood cell count, polymorphonuclear leucocytes and CRP were not suggestive of acute suppurative parotitis. Repeated serum mumps IgM was negative at the $14^{\text {th }}$ day. His parotitis improved at the $13^{\text {th }}$ day of brucellosis therapy. His cranial MR showed a meningeal enhancement on T1 (Figure 3). In case 11 , knee pain and walking disturbance emerged in the $25^{\text {th }}$ day. In her neurological examination paraparesis and tremor were detected. The spinal MRI revealed inflammation throughout the spinal canal and arachnoiditis. Case 12 had complaints of weakness, speech disturbance, double vision and imbalance for two weeks.

Figure 2: A. The patchy contrast enhancement on T1 weighted MRI scanning. B. Fusiform transverse myelitis at level C 3,4,5 on T2 weighted MRI scanning (case 5).

A

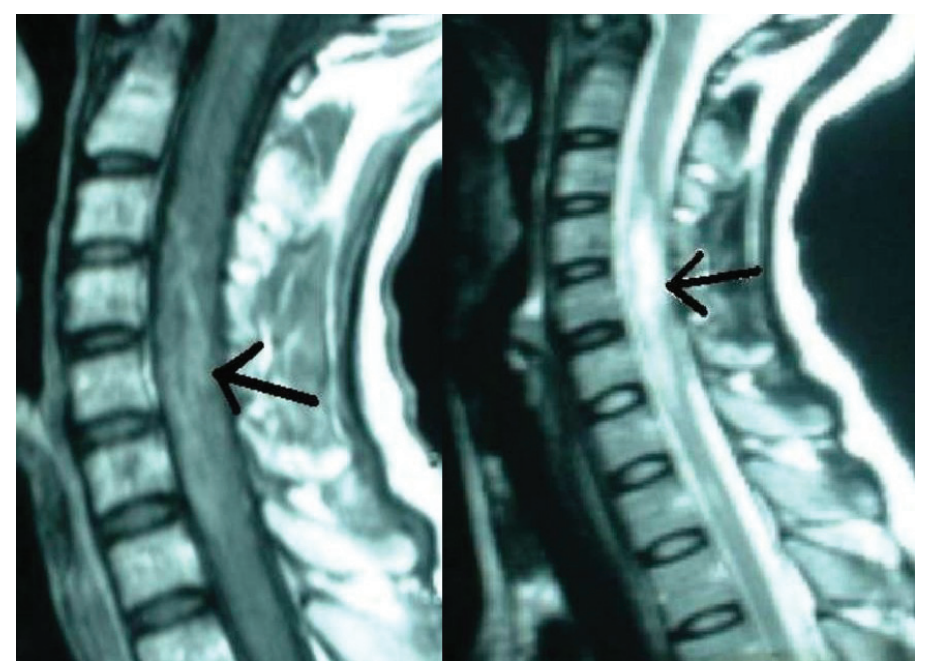

Figure 3: Wide meningeal enhancement on T1 weighted MRI scanning (case 10).

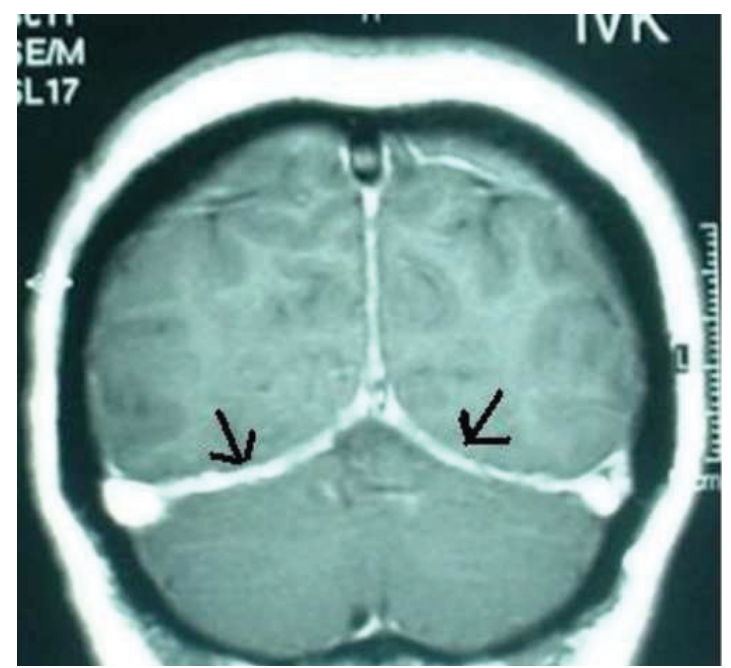


In his hospital following period, a generalized tonic-clonic seizure was observed. Cranial CT showed a hypodense nodule of about $1 \mathrm{~cm}$ diameter in the right temporal lobe (Figure 4) and MRI results showed vasculitic changes on T1 at the level of the right basal ganglia (Figure 5). Case 15 presented with aphasia and sensory loss in the upper and lower extremities. Sagital sinus thrombosis was detected in his cranial MR venography.
Fifteen patients had headache since onset of illness. Due to some patients having altered consciousness we preferred the term of meningoencephalitis. Except one (case 13), all patients had a history of using unpasteurized dairy products. In two patients the symptomatic period lasted about five and eight months. In the remaining patients duration of symptoms ranged from seven days to four months $(56.88 \pm 61.92$ days $)$. Eight patients had

Figure 4: At right temporal lobe, a granuloma with peripheral enhancement on T1 weighted MRI scanning (case 12).

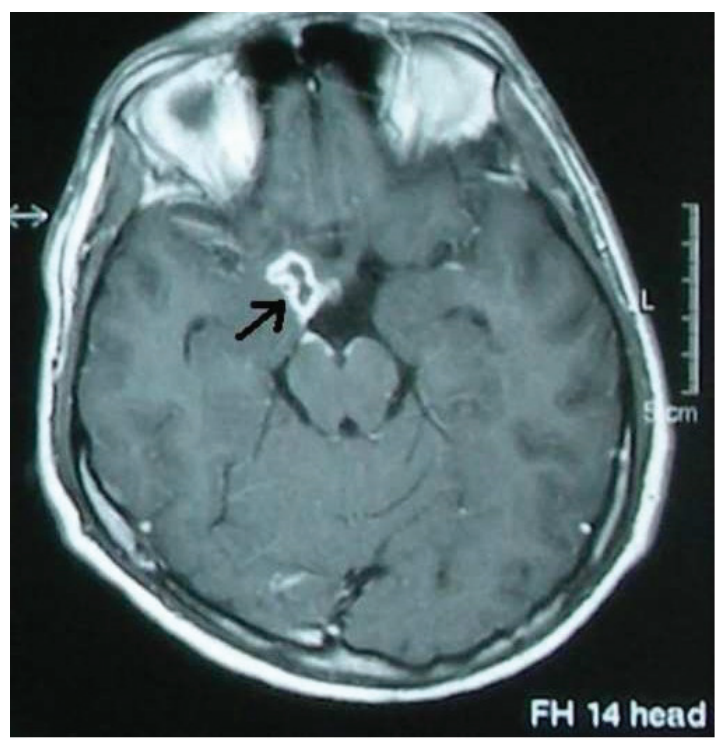

Figure 5: At level of basal ganglia, vasculitic changes on T1 weighted MRI scanning (case 12).

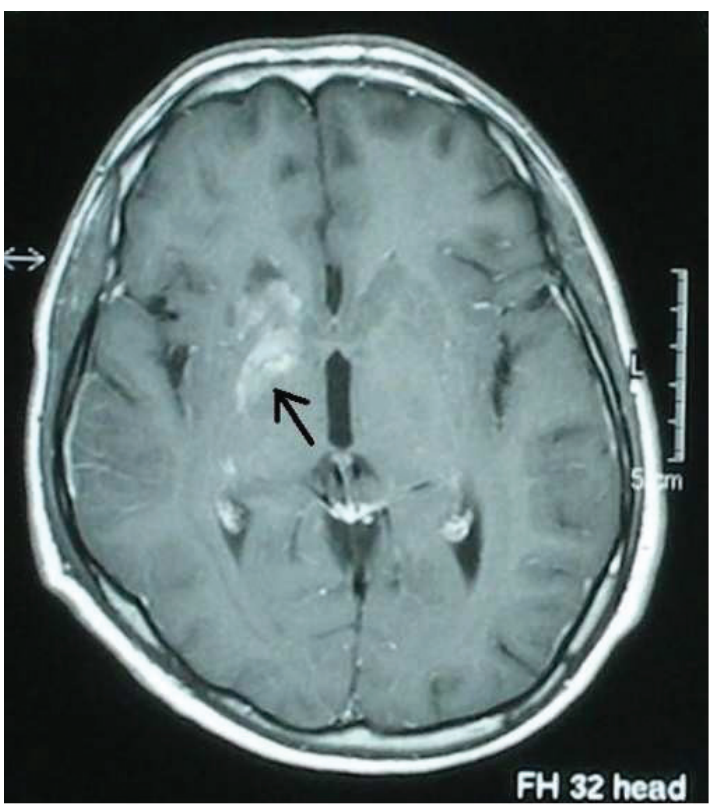


fever and five patients had signs of meningeal irritation. Cases 9, 10, 14, and 16 exhibited acute onset of meningeal syndrome. Two patients had epileptic seizures (case 2,12 ). Other additional neurological manifestations included tetraparesis, hemiparesis, cranial nerve involvements and cerebellar findings.

In two cases ( 1 and 4), patients had depressive mood and had received antidepressant for two and eight months, respectively, before admission to the hospital.

CSF analysis findings confirmed lymphocytic pleocytosis (12-750 cells $/ \mathrm{mm}^{3}$; mean $\left.250.47 \pm 246\right)$, increased CSF protein level $(82-450 \mathrm{mg} / \mathrm{dL}$; mean $171.35 \pm 97.51)$ and decreased CSF/plasma glucose ratio (18-45\%; mean $37.75 \pm 14.0)$. All patients had the diagnosis established by STA test. However in three patients (cases 10, 12, and 17) STA titer had become positive on the $24^{\text {th }}, 34^{\text {th }}$ and $20^{\text {th }}$ days after onset of the disease, respectively.

In nine patients serum cultures were positive for Brucella species. Three of the nine isolates were identified as B. melitensis. CSF immune electrophoresis was performed in patients who had white matter lesions radiologically. Oligoclonal band was negative in CSF and serum immune electrophoresis of these patients .

For each patient, the highest titer was given for standard agglutination test results in Table 1.

Chest $\mathrm{x}$-rays and backbone $\mathrm{x}$-rays were within normal limits in all patients. Neuroimaging findings were normal in six patients. The pathologies observed in neuroradiological examinations consisted of meningeal contrast enhancement, white matter changes, and vascular changes that were correlated with the clinical manifestations.

Antimicrobial therapy consisted of a combination of three drugs; doxycycline and rifampicin were used in all patients, and the third drug was one of either co-trimoxazole, ceftriaxone, or ciprofloxacin. In four patients ceftriaxone was used at onset of therapy and was changed to co-trimoxazole after three or four weeks. Ciprofloxacin was used in only two patients who had gastric intolerance and allergic reaction to co-trimoxazole. Duration of treatment was determined according to CSF response ranging from three months to one year. The patient who had pseudotumor cerebri (case 4) used acetazolamide to reduce intracranial pressure. Two patients had initially received tuberculostatics without improvement (case 10 and 12 ). In the $5^{\text {th }}$ and $7^{\text {th }}$ cases neurobrucellosis exacerbated six months due to the cessation of treatment themselves.

For the four patients (cases 5, 7, 11, 12) who had cranial nerve involvement and toxicity, prednisolone was used.

Fourteen patients recovered without any sequelae. In four patients some neurological deficits persisted. Two patients had neurosensorial deafness (cases 2, 7), one had tetraparesis (case 5), and one had hemiparesis and motor aphasia (case 6). Remarkable features of our patients are summarized in Table 2.

Table 2. Summary of various clinical features of neurobrucellosis

\begin{tabular}{lc}
\hline Features of patients & N (\%) \\
\hline Fever & $8(44.4)$ \\
\hline Headache & $15(83.3)$ \\
\hline Neck stiffness & $5(27.7)$ \\
\hline Positive MR findings & $12(66.6)$ \\
\hline Conscious alteration & $9(50)$ \\
\hline Myelitis & $3(16.6)$ \\
\hline Vascular involvement & $3(16.6)$ \\
\hline Cranial nerve involvement & $4(22.2)$ \\
\hline Paresia & $6(33.3)$ \\
\hline Sequel & $4(22.2)$ \\
\hline
\end{tabular}

\section{DISCUSSION}

Brucellosis is a common endemic infection in Turkey. Although mortality of brucellosis is rare, it continues to be a major health problem because of its morbidity.

In this infection male-to-female ratio is $2: 1$ in the literature. ${ }^{11}$ This may reflect occupational risks such as stock industry, which mostly employs males. In our series the maleto-female ratio was 1.57:1. Our patients may have acquired the disease from unpasteurized dairy product, thus it was not gender associated as reported in the literature.

Infection triggers the immune mechanism leading to a demyelinating state. ${ }^{9}$ In our first case meningoencephalitis leading to perivascular white matter lesions suggests this immunopathology. Brucellosis caused transverse myelitis in cases 5 and 7. Similar immunopathogenesis may be responsible in these cases, too. As the disease gets more chronic, the immune mechanism processes increase. ${ }^{9}$ Encephalopathy in brucellosis is always secondary to vascular involvement. Cranial nerve paralyses were seen more frequently during the acute/subacute disease course associated with diffuse CNS involvement. The acoustic nerve was described as the most frequently involved cranial nerve. ${ }^{6,711}$ Two of our patients had developed neurosensorial deafness associated with eighth nerve involvement. In three patients, $n$. abducens and $n$. facialis paralyses were found respectively. 
Our fourth case had an intracranial hypertension which developed as migraine-like headache and symptoms. Headache and psychiatric symptoms may develop due to the toxic effect of neurobrucellosis, but intracranial hypertension is a result of meningoencephalitis. Spinal arachnoiditis developed in our $11^{\text {th }}$ patient as a result of inflammation.

As noted in previous reports, the sign and symptoms of meningeal involvement are non-specific in neurobrucellosis. ${ }^{3}$ Neck stiffness occurs in less than one half of patients with meningitis. In our series, neck stiffness or Kernig's and Brudginsky's signs were observed only in five patients. The reason for the infrequent meningeal signs may be the chronicity of the infection.

Although endocarditis, hepatitis, epididymo-orchitis, spondylitis or abscess are reported in brucellosis patients, no report is found about the parotitis originating from brucellosis. ${ }^{3-18}$ Parotitis seen in our $10^{\text {th }}$ patient was not related to mumps. There was no finding suggestive of bacterial etiology. However, the parotid gland swelling emerged during the meningitis and improved with continued brucellosis therapy. We thought that parotitis was caused by brucellosis.

In our series, serum culture positivity was $50 \%$ for B. melitensis was the most commonly isolated species from neurobrucellosis cases. This may be for its being more neurotropic than other Brucella spp. CNS involvement is rare with other Brucella species. ${ }^{2,3,11}$ B. melitensis was isolated from the serum of only two of our patients. Brucella was never isolated from the CSF. We thought this was due to low bacteria density and that CSF was inoculated in nonspecific agar medium at the onset of the disease. Although ELISA is a sensitive and specific test for the diagnosis of neurobrucellosis, the most commonly used method for screening is the standard agglutination test. ${ }^{19-21}$ Antibody titers in CSF are usually lower than in serum. In some patients agglutination test is negative at the beginning of the illness, this is a well known feature in localized brucellosis and sometimes more than one serologic test is necessary before it turns out positive..$^{4-6}$ In the $9^{\text {th }}$ and $10^{\text {th }}$ cases, despite positive serum STA during the hospitalization, CSF STA turned out positive at $15^{\text {th }}$ and $24^{\text {th }}$ day of treatment, respectively. In the $12^{\text {th }}$ case, serum and CSF STA titers and Brucella IgG and IgM titers by ELISA were negative until the $34^{\text {th }}$ day.

In neurobrucellosis there are four imaging findings; normal, inflammation (abnormal enhancement), white matter changes, and vascular changes. ${ }^{22}$ In this series the four imaging presentations were observed. Although granulomatous formation results from inflammation that is relevant to infection, it is a rare manifestation in neurobrucellosis. ${ }^{23}$ This disease does not show predilection of size or location of vascular structure. Arterial and/or venous structures may be affected. ${ }^{24}$ Although in diagnosis of disease imaging results are not specific, it also contributes in the monitoring of normalization of the pathology during follow-up .
The treatment of neurobrucellosis is still controversial. ${ }^{2-6}$ There are few guidelines for the appropriate duration neurobrucellosis treatment. ${ }^{25}$ Recent reports recommend a regimen with a combination of three or four antibiotics for neurobrucellosis. ${ }^{11}$ Doxycycline is the preferred tetracycline in neurobrucellosis because its tissue and CNS penetrance is much better and it also has a longer half-life. Rifampicin and co-trimoxazole also offer a good penetration into the CSF. ${ }^{25}$ Ciprofloxacin combined (not solely ${ }^{26}$ ) with other antibiotics is as effective as the standard regimen of doxycycline and rifampicin. ${ }^{27,28}$ Ceftriaxone also offers good in vitro activity and penetration into the $\mathrm{CSF}^{29}$ Ceftriaxone alone was given to three patients at onset of disease until definite diagnosis. With this antibiotic signs and symptoms abated. Ceftriaxone can be chosen as the third antibiotic in the hospitalization period or for a patient for whom oral antibiotic could not be given. In neurobrucellosis treatment should be maintained until improvement of clinical symptoms and CSF response. Serological findings have little value to decide when to stop therapy.

In our patients treatment was generally fairly well tolerated. Co-trimoxazole caused gastric intolerance and allergic reaction in only two patient, for whom ciprofloxacin was used instead. In a previous study, gastric intolerance was reported in two of 86 patients. $^{30}$

Prognosis of neurobrucellosis varies according to clinical presentation; for example, in the meningitis group the prognosis was usually good. However, in the encephalic or spinal cord involvement, mortality is not negligible and sequelae are more frequent. In this series neurological sequelae were observed in the group with diffuse CNS involvement. It is clear that adequate duration of therapy and appropriate combination of antibiotics may prevent recurrence.

In summary, (I) brucellosis may initiate demyelinating process like as seen in the $1^{\text {st }}, 2^{\text {nd }}$, and $8^{\text {th }}$ cases; (II) brucellar meningitis may be the only sign of brucellosis, as in $9^{\text {th }}$ case or it may be first sign of brucellosis as in $10^{\text {th }}$ case; (III) the complication may be silent, part of systemic brucellosis as in $13^{\text {th }}$, $16^{\text {th }}$, and $18^{\text {th }}$ cases, and it may be overlooked; (IV) it also may be confused with tuberculous meningitis as the $11^{\text {th }}$ and $12^{\text {th }}$ cases; (V) during the first weeks of the disease, specific immune response may be delayed in serum and/or CSF like as observed in the $10^{\text {th }}, 12^{\text {th }}$, and $17^{\text {th }}$ cases; therefore, serologic tests should be repeated weekly, particularly in endemic regions.

\section{CONCLUSION}

Brucellosis continues to be a common and important health problem in developing countries and neurobrucellosis is one of the important complications of brucellosis. Neurobrucellosis may appear with different clinical manifestations and the diagnosis may be difficult. In unusual neurologic disorders brucellosis should be kept in mind especially in endemic areas. 


\section{REFERENCES}

1. URL:http://www.saglik.gov.tr/extras/istatistikler/temel2004/ tablo52.htm

2. Young J E. Brucella species. Mandell GL, Bennett JF, Dolin R(eds). In: Principles and Practice of Infectious Diseases. Churchill Livingstone USA sixth edition, 2005; 2669-2674.

3. Pappas G, Akritidis N, Bosilkovski M, Tsianos E. N Engl J Med. 2005; 352; 22: 2325-2336.

4. Colmenero JD, Reguera LM, Martos F, Sanchez-De-Mora D, Delgado $\mathrm{M}$ et al. Complication associated with Brucella melitensis infection: a study of 530 cases. Medecine 1996; 75:195-211.

5. Shakir AR, Al-Din ASN, Araj F, Lulu AR, Mousa AR et al. Clinical categories of neurobrucellosis. Brain 1987; 110:213-223.

6. Pascal J, Combarros O, Polo JM, Berciano J. Localized CNS brucellosis: report of 7 cases. Acta Neurol Scand. 1988; 78:282-289.

7. Lubani MM, Dudin KI, Araj GF, Manandhar DS. Neurobrucellosis in children. Pediatr Infect Dis J. 1989; 8:79-82.

8. Bashir R, Faan MZ, Faan EJH, Jinkins J. Nervous system brucellosis: diagnosis and treatment. Neurology 1985; 35:1576-1581.

9. Seidel G, Pardo CA, David Newman-Toker, Olivi A, Eberhart CG. Neurobrucellosis presenting as leukoencephalopathy. Arch Pathol Lab Med. 2003; 127:e374-377.

10. Al-Orainey IA, Laajam MA, Al-Aska AK, Rajapakse CN. Brucella meningitis. J Infect. 1987; 14:141-145.

11. McLean DR, Russell N, Khan MY. Neurobrucellosis: clinical and therapeutic features. Clin Infect Dis. 1992; 15:582-590.

12. Akdeniz H, Irmak H, Anlar Ö, Demiröz AP. Central nervous system brucellosis: presentation, diagnosis and treatment. J Infect. 1998; 36:297-301.

13. Namıduru M, Karaoglan I, Yılmaz M. Guillain-Barre syndrome associated with acute neurobrucellosis. Int J Clin Pract. 2003; 10:919-920.

14. Çokça F, Azap A, Meço O. Bilateral mammary abscess due to Brucella melitensis. Scand J Infect Dis 1999; 31:318-319.

15. Dakdouki GK, Araj GF, Awar GN. Buttock abscess brucellosis. Scand J Infect Dis. 2002; 34: 934-936.

16. Vajramani GV, Nagmoti MB, Patil CS. Neurobrucellosis presenting as an intra-medullary spinal cord abscess. Ann Clin Microb Antimicrob. 2005; 16:14.
17. Bucher A, Gaustad P, Pape E. Chronic neurobrucellosis due to Brucella melitensis. Scand J Infect Dis. 1990; 22:223-226.

18. Güvenç H, Kocabay K, Ökten A, Bektas S. Brucellosis in a child complicated with multiple brain abscesses. Scand J Infect Dis. 1989; 21:333-336.

19. Araj GF, Lulu AR, Saadah MA, Mousa AM, Strannegard L et al. Rapid diagnosis of central nervous system brucellosis by ELISA. J Neuroimmunol. 1986; 12:173-182.

20. Baldi PC, Araj GF, Racaro GC, Wallach JC, Fossati CA. Detection of antibodies to brucella cyoplasmic proteins in the cerebrospinal fluid of patients with neurobrucellosis. Clin Diag Lab Immunol. 1999; 6:756.

21. Sanchez-Sousa A, Torres C, Campello MG, Garcia C,Parras $\mathrm{F}$ and et al. Serological diagnosis of neurobrucellosis. J Clin Pathol. 1990:79-81.

22. Al-Sous MW, Bohlega S, Al-Kawi, Alwatban J, McLean R. Neurobrucellosis: clinical and neuroimaging correlation. Am J Neuro Radiol. 2004; 25:395.

23. Martinez-Chamorro E, Munoz A, Esparza J, Munoz MJ, Giangaspro E. Focal cerebral involvement by neurobrucellosis: pathological and MRI findings. Eur J Radiol. 2002; 43: 28-30.

24. Adaletli I, Albayram S, Gurses B, Ozer H, Yilmaz MH et al. Vasculopathic changes in the cerebral arterial system with neurobrucellosis. Am J Neuro Radiol. 2006; 27:384-386.

25. Daikos GK, Papapolyzos N, Marketos N, Mochlas S, Kastanakis $\mathrm{S}$ et al. Trimetoprim-Sulfamethoxazole in brucellosis. J Infect Dis 1973; 128 (S):731-733.

26. Doganay M, Aygen B. Use of ciprofloxacin in the treatment of brucellosis. Eur J Clin Microbiol Infect Dis. 1992; 11:74-75.

27. Agalar C, Usubütün S, Türkyılmaz R. Ciprofloxacin and rifampicin versus doxcyclinee and rifampicin in the treatment of brucellosis. Eur J Clin Microbiol Infect Dis. 1999; 18: 535-538.

28. Gogos GA, Maraziotis TG, Papadakis N, Beerman D, Siamplis DK et al. Penetration of ciprofloxacin into human cerbrospinal fluid in patients with inflamed and noninflamed meninges. Eur J Clin Microbiol Infect Dis. 1991; 10:511-514.

29. Al-Idrissi HY, Uwaydah AK, Danso KT, Qutub H, Al-Mousa MS. Ceftriaxone in the treatment of acute and subacute human brucellosis. J Int Med Research 1989; 17:363-368.

30. Pellicer T, Ariza J, Foz A, Pallarez R, Gudiol F. Specific antibodies detected during relapse of human brucellosis. J Infect Dis. 1988 ; 157:918-24. 